N

2050

A7A6 bn Centrale des Arts Décoratifs

1905

CHM

\title{
GUIDE SOMMAIRE
}

\author{
A TRAVERS LE
}

Musée des Arts Décoratifs

PALAIS DU LOUVRE = PAVILLON DE MARSAN

107, RUE DE RIVOLI

('n lace la rue de l'Échelle)

PARIS

IN PRIMERIE GÉNÉRALE LAHURE

9, RUE DE Fleurus, y

$100 . \overline{5}$ 

les ouvrages les meilleurs des artistes industriels contemporains. La création de ce musée fut rendue possible par l'autorisation, reçue en $\mathrm{i} 882$, d'émettre une loterie: la loterie produisit près de 6 millions de francs, et ce sont les intérêts de cette somme qui permirent à la Société de constituer le Musée qui devait couronner son œuvre.

Le Musée des Arts décoratifs s'ouvrit au Palais de l'Industrie et il s'accrut rapidement par des achats, par des dons et par des legs; il rendait des services que savaient apprécier les nombreux ouvriers d'art qui y renaient dessiner ou modeler, quand la démolition du Palais de l'Industrie l'obligea à mettre ses collections en caisse et à chercher pour elles un autre local. L'État, qui s'était engagé envers l'Union Centrale des Arts décoratifs à lui en trouver un, consentit, après de longues négociations menées par le président, M. Georges Berger, à lui céder le Pavillon de Marsan, au Louvre, 'et c'est là que le Musée est aujourd'hui ouvert au public; mais le don n'a pas été gratuit: en échange du palais que l'Union Centrale devait aménager à ses frais, elle a dû s'engager à faire remise gratuitement à l'État, au bout de quinze ans, de toutes les collections qu'elle y aurait installées. Dès maintenant, grâce à ses acquisitions, grâce aux libéralités nombreuses des amateurs et des artistes, grâce au legs qu'Émile Peyre lui a fait de son admirable collection, le Musée se présente d'une façon digne du toit qui l'abrite, et digne de Paris: pendant les quinze ans que l'Ĺnion Centrale continucral de l'administrer, elle l'enrichira encore et elle espère que le public l'aidera, comme il l'a fait jusqu ici, dans la réaltsation de l'œuvre artistique et patriotique qu'elle al cntreprise, et qui consiste à célébrer le passé de nos industries d'art, à en faire reconnaitre l'effort présent et à leur préparer un glorieux avenir. 


\section{RÈGLEMENT}

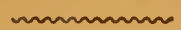

\section{I. - Musée des Arts décoratifs}

\section{Le Musée des Arts Décoratifs est ouvert tous les jours :}

Du I.5 Avril au I $^{\text {r }}$ Octobre : de 10 h. du matin a 5 h. du soir.

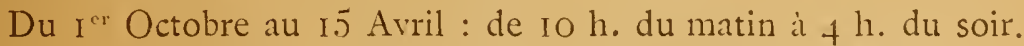
(Le Lundi, le public n'est admis qu'à partir de Midi.)

L'entrée est gratuite chaque dimanche.

Le prix d'entrée est fixé à I franc par personne les jours de semaine; il est abaissé a o fr. 50 les jours fériés autres que les dimanches.

Le service des tourniquets d'entrie cesse une demi-heure avant la fermeture du Musée qui est annoncée, un quart d'heure d'avance, p.ur les gardiens préposés à l'éracuation des salles.

A côté des tourniquets d'entrée sont ménagés des guichets par lesquels pénètrent les porteurs de cartes donnant l'entrée gratuite, notamment les Sociétaires de l'Union Centrale des Arts Décoratifs et les Membres de ses Comnilissions consultatives.

Des cartes d'abonnement annuel, renouvelables et strictement fersonnelles, sont tenues au prix de 10 francs a la disposition des femmes et des enfants des Membres sociétaires.

Le Musée des Arts Décoratifs est une anvre d'initiative privée qui se suffit à elle-mêtric par ses propres ressources; il ne reçoit ancune subvention. des Pouvoirs publics. Ses recettes sont intégralement consacrées à son entretien, à son développement et à l'enricluissement de ses Collections.

On ne sanrait donc s'étonner que la Société de l'Union Centrale des Arts Dícoratifs use de la faculté que lui confére l'art. 7 de la convention légale du 3 Mars 189-, en faisant payer un droit d'entrée cloque jour, sanf le Dimanclue.

Les visiteurs doivent être conzaincus qu'en acquittant le prix de leur entrée, ils versent une obole à un établissement national d'enseignement de l'Art appliqué.

Faire sous cette forme un appel à ceux qui voient dans l'Art une partie de l'bonneur et de la fortnue du pay's, c'est inroquer le patriotisme de tous les bons Franciais. 
Des tickets d'entrée gratuite pour une journéc peuvent être accordés.

Les guichets d'entrée gratuite servent également à la sortie des visiteur's.

Il est perçu un droit de o fr. Io de la part de toute persomne qui fait usage de l'ascenseur pour monter à un étage quelconque, à partir de n'importe quel point de départ. Un droit de o fr. Io par personne est dû pour toute descente

Aucune carte d'entrée au Musée ne donne le droit d'user gratuitement de l'ascenseur.

Il est interdit de toucher aux objets exposés.

Des cartes spécialıs temporaires et renouvelables peurent être délivrées, sur leur demande dûment justifiée, aux artistes qui exécutent des copies à la main d'objets exposés. Les personnes autorisées ì cet effet derront fournir leur chevalet et leur siège ; elles pourront confier ce matériel aux gardiens des salles. Afin d'éviter des encombrements gênants pour la circulation des visiteurs, deux personnes ne pourront dessiner à la fois d'après le même modèle.

Les photographes professionnels faisant usage d'appareils supportés par des pieds seront passibles du prix d'entrée ordinaire au Musée; ils devront se munir d'une autorisation. Ils ne seront admis à opérer que le matin, jusqu’à midi.

Les copistes et les photographes professionnels ne seront pas admis à travailler le dimanche et les jours fériés.

L'administration se réserve le droit d'interdire la présence des photographes et des copistes chaque fois que les besoins du service intérieur exigeront cette interdiction.

Un vestiaire est ouvert à l'entrée du Musée. Il est facultatif, sanf pour les paquets qui doivent, obligatoirement, y être déposés.

Des plaques indicatrices sont placées en évidence dans toutes les parties du Musée, de façon à permettre aux visiteurs de s'y diriger.

Des troncs au profit des ouvriers et apprentis d'art du département de la Seine seront répartis dans le Musée.

\section{II. - Bibliothèque des Arts décoratifs}

\section{La Bibliothèque de l'Union Centrale des Arts Déco-} ratifs est publique.

Elle est ouverte gratuitement tous les jours non fériés. de Io li. du matin ì 5 h. I/2, et, le soir, de ? h. 1/2 à in heures.

Elle est fermice chaque annéc du $\mathrm{I}^{\text {rr }}$ all 20 Août. 
Les lecteurs sont tenus de s'adresser aux Bibliothécaires ou aux Agents de service pour les demandes de livres et de remplir à cet effet un bulletin signé de leur nom. Ce bulletin indiquera leur adresse et leur qualité.

Aucun livre, dessin ou document quelconque, ne peut être prêté pour être emporté en vue d'un travail à domicile.

Les lecteurs sont responsables des déchirures ou détériorations dont ils sont reconnus être les auteurs.

Les lecteurs sont expressément invités à placer leur pardessus et chapeau dans les filets tendus à cet effet au-dessous des tables et pupitres. Des porte-cannes et porte-parapluies sont mis d'autre part à leur disposition.

L'Administration n'est pas responsable des objets disparus.

Les conversations à haute roix sont interdites.

Toute personne qui provoque un désordre ou se fait remarquer par sa mauvaise tenue est invitée à sortir.

La fréquentation gratuite de la Bibliothèque n'affranchit pas les lecteurs de l'acquittement du prix d'entrée dans le Musée, à moins qu'ils ne soient détenteurs de cartes persomnelles délivrées à un titre justifié et prévu.

\section{III. - Bureaux d'Administration}

Les Bureaux d'administration de l'Union Gentrale des Arts Décoratifs sont ouverts chaque jour non férié de Io heures du matin à midi et de 2 heures de l'après-midi à 5 heures du soir.

Les Bureaux de la Société des Amis du Louvre sont contigus à ceux de l'administration de l'Union Centrale des Arts Décoratifs. 


\section{DIRECTION}

L'entrée du Musée des Arts décoratifs, de la Bibliothéque des Arts décoratifs et des Bureaux de la Société a lieu par la porte située $\mathrm{n}^{0}$ Io rue de Rivoli (en face de la rue de l'Échelle). Cette porte donne accès dans. le restibule principal ou "Guichet de l'Échelle ".

A droite de l'extrémité du "Guichet de l'Échelle ", du côté des jardins du Carrousel, s'ouvre la salle de lecture de la Bibliothèque.

Au milieu du guichet, à gauche et à droite, deux grandes arcades se font vis-à-vis. L'arcade de droite donne accès dans la grande nef du Musée (Sąlle I08). Celle-ci est précédée d'un vestibule à droite duquel, du côté de la rue de Rivoli, se trouve le départ de l'Escalier Lefuel (C) qui conduit au I $^{\text {er }}$ étage; en face de cet escalier, du côté du Carrousel, le visiteur rencontre l'Escalier de la Bibliothèque (B) qui mène à tous les étages, de même que l'Escalier de Marsan (D), situé à l'extrémité droite de la grande nef, du côté de la rue de Rivoli.

Un ascenseur est installé près de l'Escalier de Marsan.

L'arcade de gauche donne accès à un autre vestibule sur lequel s'ouvrent, au premier rez-de-chaussée, les Bureaux de l'Administration. De ce vestibule part un escalier dit Escalier de Rohan (A), qui conduit aux $B u$ reaux de la Conservation du Musée, situés au grand rezde-chaussée, puis aux salles d'exposition des deux étages supérieurs.

Au rez-de-chaussée du Musée, dans les salles donnant sur la rue de Rivoli et sur le jardin des Tuileries, sont installées (Salles I Io à I26 et Salle 123) les collections d'Art moderne ( $x_{1} x^{\mathrm{e}}$ siècle) et, provisoirement, dans les 
salles donnant sur le jardin du Carrousel, les collections d'art de lorient (Salles I1.3, I 15. I I7) et de l'ExtrêmeOrient (Salles I I9 et I2 I), ces dernières collections seront transférées plus tard dans le Grand-Comble, actuellement en roie d'achèrement, et les salles demeurées libres au rez-de-chaussée seront consacrées, ainsi que la grande nef. il des expositions temporaires.

Au premier étage sont installées, dans les salles donnant sur le jardin du Carrousel, à gauche du Guichet de l'Échelle, les collections d'Art gothique (Salles 20I-207) et, dans les salles donnant sur la rue de Riroli, les collections d'art de la Renaissance (Salles 206-226). Dans les salles donnant sur le jardin des Tuileries sont les collections d'art de l'époque de Louis XIV (Salles 228-2.34) et, dans les salles en retour sur le Carrousel, celles de l'époque de Louis XV (Salles 209 à 221 ).

A l'entresol du premier étage sont installées, dans les salles donnant sur le jardin du Carrousel, à gauche dı (iuichet de l'Échelle, les collections d'art italien (Salles $25 \mathrm{I}-257$ ) et dans les salles correspondantes, sur la rue de Rivoli, les collections d'art allemand (Salle 256) et espagnol (Salles $2 \lesssim 8-260)$. Dans les salles donnant sur a rue de Rivoli sont les collections d'étoffes (Salles 2622?2) et dans celles donnant sur le Carrousel sont la collection de ferronnerie (Salle 269) prètée par M. Le Secq des Tournelles et les collections d'art de l'époque de Louis XVI (Salles 250-267).

Le second étage ou grand-comble sera aménagé ultérieurement. 

GRAND REZ-DE-CHAUSSÉE 



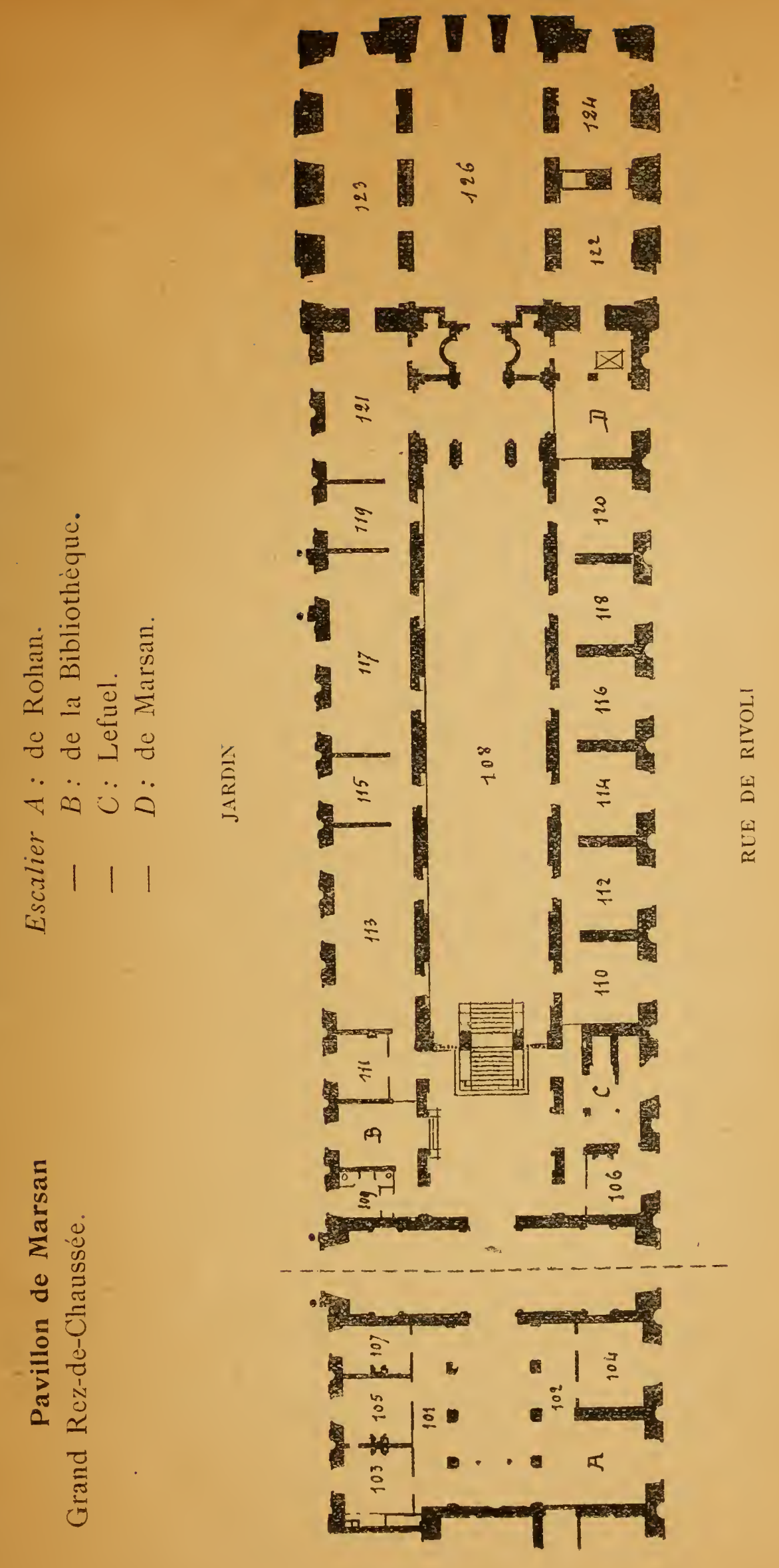




\section{GUICHET DE L'ÉCHELLE}

Vases de Sivres. - Modèles des Groupes de CarrierBelleuse, pour l'Opéra. - Grilles de Robert.

\section{VESTIBULE DE GAUCHE}

Groupes en terre cuite dans le style de Coysevox (don de M. Fitz Henry). - Buste d'Edouard André, ancien président de l'Union centrale, par MIme Ed. André.

\section{VESTIBULE DE DROITE}

Modèles des Groupes de Frémiet pour le Muséum d'histoire naturelle.

\section{Salle 106}

Reproductions galvaniques de la maison Christofle d'orfèvrerie du Moyen Age, de la Renaissance, des xrıI et xvin ${ }^{\mathrm{e}}$ siècles.

\section{Salle 110}

\section{SOUVENIRS DE NAPOLÉON $\left.\right|^{\mathrm{c}}$}

A u.r murs: Dessins pour des épées, des sabres et dés ceinturons destinés à Napoléon $I^{\text {er }}$ et à sa famille, par Biennais. - Frise italienne (legs Peyre). - Cartons pour des soieries de Lyon.

Vitrine A : Robes de femme; bonnets de l'Impératrice Marie-Louise.

Vitrine B : Habits de cour de Napoléon Ier, ayant figuré jadis au Mlusée des Souverains. 
Salle 112

EMPIRE

A u.r murs: Dessins originaux de Huet, destinés ì servir de modèles à la manufacture de toiles de Jouy (don de M. Barbet de Jouy). - Panneaux peints décorés d'arabesques. - Panneau brodé à fond bleu et modèles de broderies pour robes et habits de cour. - L'Intéricur Villageois, par Roehn (legs Peyre). - Lutte de Mincrve et d'Arachné, lavis par Chaudet (don Maciet). - Vaudoyer, Etudes d'architecture (don Vaudoyer). - Frisesitaliennes (legs Peyre).

Meubles : Lits, secrétaire, commode en acajou ornés de bronzes (legs de $M^{\text {me }}$ Dècle, don de $M^{\text {lle }}$ Fournier).

\section{Salle 114 \\ RESTAURATION}

A ux murs : Peinture en camaieu figurant un Sacrifice d Mercure; dessins originaux pour servir de modèles à la manufacture de toiles de Jouy, par Huet (le Chevalier à la Croisade), par H. Vernet (Scènes de chasse), etc. (don Barbet de Jouy). - Portraits par Boilly et de l'atelier de Drolling. - Eugène Lamy, le Hall du chàtcau de Ferrières. - Esquisse de Galland, pour le Henri IV de la galerie d'Apollon.

Meubles : Mobilier de la chambre à coucher du savant Magendic, en acajou clair et cuivres (legs de $M^{110}$ de SaintMaurice); vases de porphyre (legs de M"me de Noas).

Vitrine: Surtout de table de Charles X par Odiot (legs Audéoud).

\section{Salle 116.}

\section{ÉTUDES DE FLEURS}

Aux murs : Claudius Popelin, Paravent décoré de roses, et Éventails (legs de S. A. I. la princesse Mathilde). 
- Études de fleurs, par Chabal-J)ussurgey, (ialland. Poirier, ete. - Panneaux décoratifs de P.-V. Cialland. - Diane, lapisserie des Gubelins sur les cartons de Machard.

Vitrine: Verrerie, par Rousseau, Léveillé, Brocard, etc.

Vitrine ronde: Émaux, par Claudius Popelin (legs de S. A. I. la princesse Mathilde).

\section{Salle 118 \\ CÉRAMIQUES ÉTRANGÈRES}

A ux murs: Panneaux décoratifs de Steinlen et de Chéret, projet de vitrail pour l'École de pharmacie de Paris, par Besnard. - Panneau de Baudry, exécuté par Decl. - Dessins de Charlemont pour la décoration du Burgtheater de Vienne. - Plateaux de bronze ciselés par Mme L. Croce. - Buste de Rurik, par M. Antokolsky, en céritmique de Deck.

Vitrine A : Porcelaines de la manufacture royale de Copenhague : assiettes, rases, animaux. - Porcelaine de Groendael, de Delft et de Rosendael.

Vitrine B : Céramiques : anglaises, de Doulton et Minton; allemandes, des manufactures royales de Berlin et de Meissen, de MM. le professeur Laüger, Mutz, Saint-Lerche; italiennes, de Ginori: autrichiennes, de M. Carrier; américaines, de Crueby.

Vitrine C: Verres de Bohême.

Vitrines $\mathbf{D}$ : Reliures anglaises.-Nédailles par Kautsch.

\section{Salle 120 \\ CÉRAMIQUE}

Aux murs : Décoration d'un Cabinet de bains, par Chatplet, d'après le modèle de Coulon, sculpteur. - La Cirramique, groupe exécuté à Sèvres, par Coutan (réduction du monument du square Saint-Germain-des-Prés); la Forêt- 
Vierge et Perroquels, panneaux de Parvillée; Paysage et Rhododendrons, par Utzschneider (Sarreguemines); Flcurs, par Muller. Pcinture décorative, par Steinlen.

\section{ESCALIER DE MARSAN}

Palier du rez-de-chaussée.

Vases, par Chaplet; - Panneaux de LechevallierCheviguard, pour le chàteau de Saint-Roch.

\section{Salle 122 \\ SECOND EMPIRE}

A ux murs: Tapisseries de Beauvais, Fables de La Fontaine; Fleurs, par Tony Faivre. - Panneaux décoratifs : l'Industric, par Erhmann; La Charmeuse de serpents, par Froment; Projets pour la décoration d'un salon des Tuileries, par Chaplin (don de S. M. l'Impératrice Eugénie), dessins par CarrierBelleuse, Carpeaux, Jacquemart, Lièvre, etc.

Pendule en bronze doré, par Constant-Sevin (exécutée par Barbedienne), et une autre sur les dessins de Gustave Doré.

Vitrine A : Maquettes de Carpeaux (projets de statues, étude d'après le Prince Impérial); Bronzes de Barye.

Vitrine B : Céramique: imitations de Bernard Palissy, par Avisseau et Pull; d'Orient, par Deck et Parvillée; dc Rouen, par Boulanger, etc.

Vitrine $\mathbf{C}$ : Orfèvrerie : Pièces du service de table des Tuileries, exécutées par Christofle; coupes et aiguières dans le style antique, par Constant-Sevin; poisson, par Varangoz; vases, par Levillain; calice, par Armand Calliat.

Bibliothèque en ébène, par Fourdinois, contenant des rcliures, dessinées par Rossigneux. 


\section{Salle 124 \\ VERS $1880-90$}

Aux murs: Sept cartons de vitraux d'Albert Besnard, dessinés pour l'école de Pharmacie de Paris, et représentant des animaux dans des paysages; dessins, par Grasset, Sandier, Couty, etc.; panneaux de céramique de Muller, etc.

Vitraux exécutés sur les cartons de Grasset et Albert Besnard.

Vitrines $\mathbf{A}$ et $\mathbf{B}$, dessinées par Grasset, contenant des céramiques de Rousseau (d'après les dessins de Braquemond), Collinot (d'après les dessins de Lepic), Deck, Milet, Bouvier, Cazin, Chaplet, Delaherche, Dammouse, Massier, T. Doat, etc.

Vitrine $\mathrm{C}$, faïences, par $\mathrm{M}^{\mathrm{me}}$ Adolphe Moreau (don Et. Moreau-Nélaton)

Vitrine $\mathrm{D}$, dessinée par Sandier, contenant des étains, par Brateau.

\section{Salle 126}

\section{SALLE DE SĖVRES}

A u.t murs: Réduction de la Frise exécutée à Sèvres en Igoo pour le Grand Palais, sur les dessins de Blanc. Cartons des panneaux décoratifs de P.-V. Galland, exécutés par les Gobelins pour un salon de l'Élysée. Porte, dessinée par Sédille, exécutée, pour la boiserie, par Fourdinois; pour l'émail, par Roussel; pour les bronzes, par Allard. - Autre Porte exécutée par Fourdinois sur les dessins de Chéret. - Vases de Sèvres. - Panneaux de Deck.

Vitrine A. Produits de la Manufacture nationale de Sèvres sous le Second Empire.

Vitrine B. De 1870 à i 880 environ. 
Vitrine C. De 1880 it 1895 environ.

Vitrine D. En Igoo.

A u centre. - Vitrine, par Edme Couty, en marqueterie contenant des verreries de Gallé, Rousseau, Brocard, etc.

- Tapis de Jorrand.

Surtout de table en bronze argenté, exécuté en 1855 pour les Tuileries par la maison Christofle sur lẹ dessins de F. Gilbert.

Départs de rampes d'escalier, l'une exécutée pour le chàteau de Chantilly, par Moreau, sur les dessins de Daumet (don Moreau), l'autre exécutée par Majorelle, pour un hôtel de Nancy.

\section{Salle 123}

\section{GRAND SALON MODERNE}

Le Grand Salon Moderne est la restitution de celui que l'Union centrale des Arts Décoratifs avait installé à l'Exposition de Igoo; l'architecte en est M. Georges Hœntschel; les étoffes ont été dessinées par M. Karbousky. Il est encadré de deux petites salles qui lui servent, en quelque façon, d'antichambre.

Peinture: L'Ile heureuse, par Albert Besnard; dessus de porte du même; panneaux décoratifs par Aman Jean; l'Harmonie, par Henri Martin.

Meubles: Burcau, par Janssen; casicr à musique, par Madeleine et Becker; table, par Herold; guéridon, par Majorelle; dressoir, par Barbedienne; vitrine, par de Feure.

Céramique : Grès, par Carriès, Delaherche, Dalpeyrat, Bigot, Dammousc, Hœntschel, Moreau-Nélaton, Jeanneney, Lachenal, etc. Porcelaines flammées, par Chaplet.

Émaux, par Thesmar, Grandhomme, Hirtz, Garnier, Naudot.

Verrerie, pár Émile Gallé, Rousseau, Léveillé, Daum. - Páles de verre, par Cross et Dammouse. 
Orfèvrerie : Goblet d'or à émaux translucides, exécuté par Falize, sur les dessins d'Olivier Merson; pièces diverses, par Christofle, Cardeilhac, Peureux, Mouchon, Bonvalet, Gaillard, Lelievre, Becker, Risler.

Bijoux: Montre, peignes, pectoral, par Lalique; peignes, par Vever; bracelcts, par Falize, Gaillard, Monod, Brateau.

Dentelles : Napperon exécuté par Lefébure sur les dessins de Corroyer.

Ivoire : LLa Pai.r du Foyer par Dampt; statuctte, par Barrias, exécutée par Susse frères.

\section{Salle 121}

\section{JAPON}

A u.r murs: Grand paravent en laque dit de Coromandel (xvII ${ }^{\mathrm{e}}$ siècle). - Broderies japonaises : panneau brodé du XII siècle figurant le Deva Nitten, tenant dans şa main le disque du soleil quil personnifie (don Hayashi); paravent en soie chamois brodé de touffes de fleurs ( $\mathrm{xI}^{\mathrm{e}}$ siècle); tapis de soie chinois. - Estampes japonaises du Xvme siecle (don H. Vever. R. Kœchlin et A. Rouart). - Tapis d'Orient (don Maciet).

Vitrines A et D. Étoffes japonaises du xve au xvir ${ }^{e}$ siècle : yclours figurant des éléphants stylisés; soieries il papillons, éventails, etc. Vanneries.

Vitrine B. Porcelaines du Japon : Plats de Koutani (xvIII ${ }^{e}$ siècle; don Hayashi); assiettes de Nabeshimal (xvir ${ }^{e}$ siècle). - Bronzes de la Chine et du Japon.

Vitrine C. Laques japonais (xvure siècle; don Léon Dru).

Vitrine E. Grès japonais des diverses fabriques: Vases à eau, bols, pots à thé (Tchärés), vases à fleurs ( $\mathrm{xvI}^{\mathrm{e}}$ au $\mathrm{xIX}^{\mathrm{c}}$ siècle). - Statuettes par Mme Koren (xix ${ }^{\mathrm{e}}$ siècle; en partie don Hayashi.)

Vitrines-tables $\mathbf{F}$ et $\mathbf{G}$. Peignes et gardes de sabres. 


\section{Salle 119 \\ CHINE}

A ux murs: Velours et broderies de Chine; tapis d'Orient.

Vitrine A. Porcelaine de Chine bleue et blanche (xvil$\mathrm{xix}^{\mathrm{e}}$ siècle).

Vitrine B. Porcelaine de Chine polychrome (xvit" et xIx siècles; en partie dons Gasnault et Rochard).

Vitrines C et D. Émaux cloisonnés de la Chine ( $\mathrm{XV}^{\mathrm{r}}$ xvı ${ }^{e}$ siècle; don Rochard); du mème don, au milieu de la salle, Brûle-Parfum et Vasque en émail cloisonné.

Vitrine E. Verreries de la Chine (xvie-xixe siècle).

Salle 117

ORIENT

Aux murs: Tapis persan du xrie siècle à fond jaune. décoré de personnages et de faures au milieu de jardins fleuris (don Maciet); tapis de Perse et d'Asie Mineure. Carreaux de Céramique de Syrie (à tiges de fleurs sortant de rases bleus), d'Asie Mineure et de Constantinople. Pièce de suspension en fer ourragé et damasquiné (xrve siècle).

Vitrines A et D. Velours et soieries de fabrication orientale ( $\mathbf{x} \mathbf{v}^{\mathrm{e}}$ et $\mathrm{xvI}^{\mathrm{e}}$ siècles).

Vitrine C. Assiettes en faïence dite de Rhodes (xvir ${ }^{p}$ et xviri ${ }^{\mathrm{e}}$ siècles), legs de Mme de Tournières-Jouassain.

Vitrine centrale $\mathbf{E}$. Cuivres et armes orientales. Deux aiguières décorées d'inscriptions ( $x^{\mathrm{e}}$ siècle); casques incrustés d'argent ( $\mathrm{xv}^{\mathrm{e}}$ et $\mathrm{xvi}^{\mathrm{e}}$ siècles); cuirasses, casques, plaques d'armures, étriers incrustés d'or, d'argent et de cuivre.

Vilrines-tables $\mathbf{F}$ et $\mathbf{G}$. Fragment de plaques de revêtement en plâtre estampé provenant de Koniah (xII" siècle, Asie Mineure; don R. Koechlin). Fragments de plaques de 
revêtement de l'Asie centrale en faïence émaillée (don H. Lirafft). - Velours et soieries.

Meuble toumant contenant des héliogravures daprès les photographies de M. Krafft. figurant des vues et des types de l'Asie centrale.

\section{Salle 115 \\ PERSE}

A u.x murs : Tapis persan à rehauts d'argent, décoré de fleurs, de rinceaux et d'inscriptions ( $x v^{e}$ siècle). - Carreaux de faïence persans mordorés à reflets, décor d'inscriptions en bleu (xir ${ }^{e}$ siècle). - Miniatures persanes et indoues ( $\mathrm{xvI}^{\mathrm{e}}-\mathrm{XvII}^{\mathrm{e}}$ siècle). - Soies brochées persanes (xvi ${ }^{e}-$ xvII $^{e}$ siècle). - Panneaux de bois incrustés d'ivoire (Egypte, xvI $^{\mathrm{e}}$ siècle).

Vitrine A. Céramique persane : Plaque de revêtement mordorée à reflets $\left(\mathrm{xuI}^{\mathrm{e}}\right.$ siècle); bouteilles et bols mordorés et bleus; plats à décor imité de la porcelaine de Chine (Xvirxvire siècle).

Vitrines-tables $\mathbf{B}$ et $\mathbf{C}$. Carreaux de revêtement de céramique en forme d'étoiles, mordorés et bleus, à décor de personnages, d'animaux, d'inscriptions ou de rinceaux $\left(\mathrm{XII}^{\mathrm{e}}\right.$-XVI ${ }^{\mathrm{e}}$ siècle).

\section{Salle 113 \\ ORIENT}

A ux murs : Tapis persan du xvie siècle décoré de cavaliers et de fauves sur fond noir (don Maciet): tapis persans et de l'Asie Mineure. Carreaux de céramique d'Asie Mineure et de Constantinople ( $\mathrm{xvI}^{\mathrm{e}}-\mathrm{xvII}^{\mathrm{n}}$ siècle).

Vitrine A. Céramique de Syrie et d'Asie Mineure ; bol mordoré à reflets de Rakka (Euphrate. xiv siècle) : plat at rinceaux bleu et blanc du lioutaïch (xve siècle): plat de 
Damas, à palmettes bleu sur bleu : bouteille dite de Rhodes (xvII ${ }^{\mathrm{e}}$ siècle; don Gagneau).

Vitrines $\mathbf{B}$ et $\mathbf{C}$. Velours et soieries de fabrication orientale $\left(\mathrm{xv}^{\mathrm{e}}-\mathrm{xvI}^{\mathrm{e}}\right.$ siècle).

Vitrine D. Céramique de Syrie et d'Asie Mineure : assiette de Damas à décor de roses bleu sur bleu ( $\mathrm{xvI}^{\circ}$ siécle); plat de Damas à décor de tulipes ( $\mathrm{xvr}^{\mathrm{C}}$ siècle).

Vitrine centrale $\mathbf{E}$. Cuivres incrustés d'argent : chandelier de Mossoul à sujets chrétiens' (xir" siècle); bassins (xive siècle). - Verrerie : lampe de mosquée, Vase du XIve siècle (Égypte ou Syrie) en verre blanc émaillé. Ivoire : coffret hispano-arabe diaté de .555 de l'hégire $(95.5$ de l'ère chrétienne).

Vitrines-tables $\mathbf{F}$ et $\mathbf{G}$. Fragments de céramique trouvés au vieux Caire (xille-xvil ${ }^{\mathrm{e}}$ siècle). - Reliures persanes (xvir-xix siècle). 



\section{PREMIER ÉTAGE}




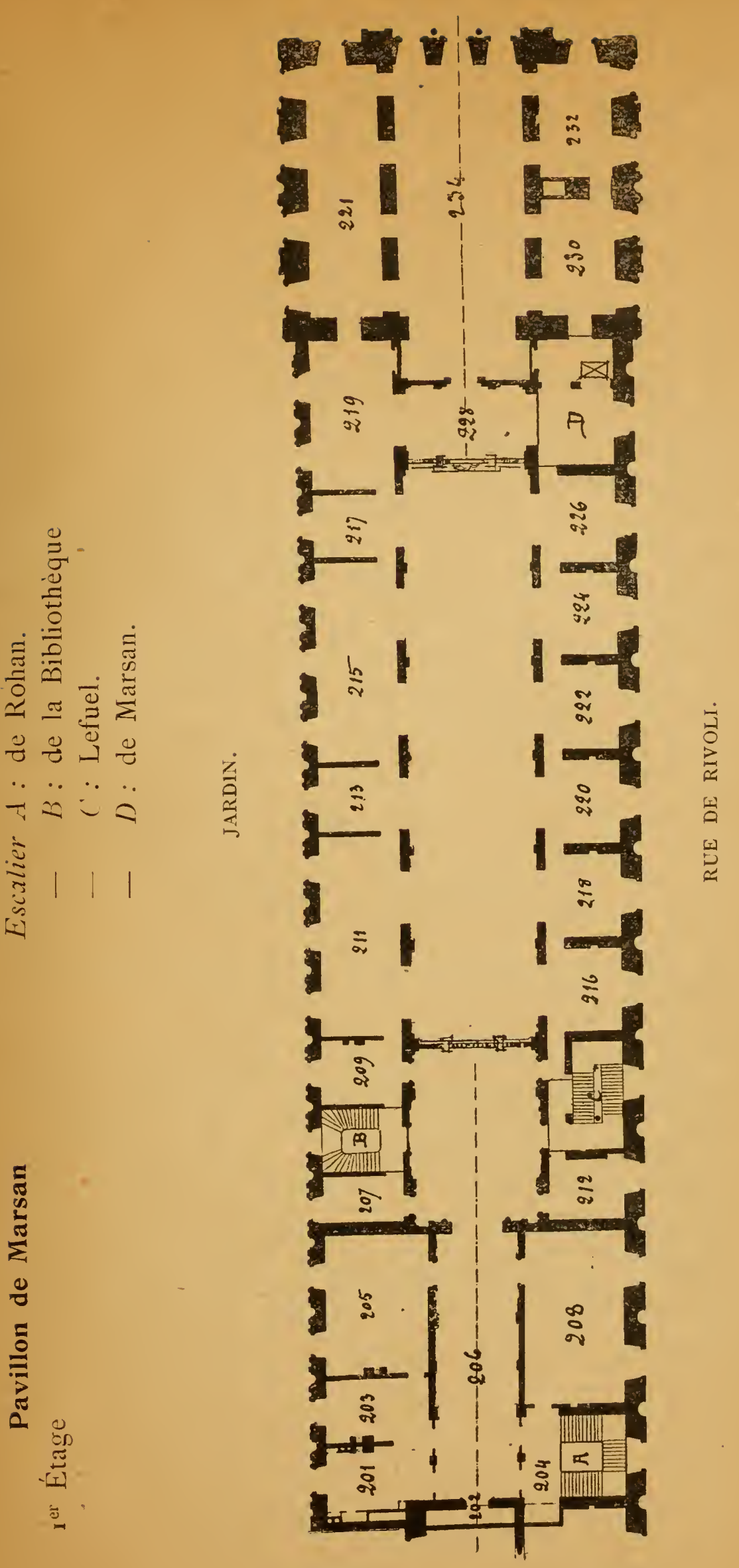


\section{Salle 206 (Galerie de Pierre)}

\section{GOTHIQUE, FRANÇAIS}

A u.r murs: Tapisseries, trois tentures à sujets dallégorics morales (legs Perre): scine de Vendanges (don Maciet); Sacre d'un évéque (don Perre).

Sculptures : Vierge et enfant (xiv siècle: pierre peinte, (don Naciet): Roi Mage (ڤ) à genoux (Bourgogne, fin $\mathrm{xV}^{\mathrm{e}}$ siècle: (don Maciet); Vierge à l'enfant (xve siècle, legs Peyre); Vierges, Saints et Saintes des xIV", $\mathrm{xV}^{\prime \prime}$ et du commencement du Xvi siècle.

Lutrin du $\mathrm{xV}^{\mathrm{e}}$ siècle (bois) en forme daigle, supporté par un pied orné de statuettes et flanqué dares-boutants. Puits en pierre (Flandre, xir siècle).

\section{Salle 201}

\section{GOTHIQUE FRANÇAIS}

La plupart des objets exposés dans cette salle font partie du legs Peyre.

A ux murs: Tenture de cinq tapis, du commencement du $\mathrm{xV}^{\mathrm{e}}$ siècle, figurant des scènes tirées d'un roman indéterminé : jeunes gens et jeunes femmes sur fond de paysage.

Sculptures : ange en bois peint provenant de Saint Germer (Oise) (xmi siècle, don Maciet): $S^{\mathrm{e}}$-Jean en hois (xive siècle).

Meubles : Coffre en bois à ferrures du xinle siècle; devants de coffres, deux crédences et deux stalles du $\mathrm{xr}^{*}$ siecle. 
Salle 203

\section{GOTHIQUE FRANÇAIS}

La plupart des objets exposés dans cette salle, excepté les tapisseries, qui ont été données par M. Maciet, font partie du legs Peyre.

A u.x murs : Tapisseries (don Haciet), Le Christ arec les Vertus; La justice divine (fin du $\mathrm{xv}^{e}$ siècle); personnages en costume du milieu du $\mathrm{xv}^{\mathrm{e}}$ siècle.

Sculptures: Vierge assise, bois, xive siecle (don R. Kiøchlin); Vierge debout (bois, xIve siècle): Vierge debout (bois xmi siècle, don Maciet); Christ tenant le globe (bois, $\mathrm{xr}^{\mathrm{e}}$ siècle); Diacre (statuette pierre, xille siècle) et Pleurant (pierre, école bourguignonne $\mathrm{xv}^{\mathrm{e}}$ siècle, dons Maciet). - Retable en bois peint et doré figurant le Portement de croix, la Crucifixion et la Déposition de croix (Flandres, commencement du $\mathrm{xrr}^{\mathrm{e}}$ siècle.

Meubles: Trois crédences et coffre du xve siecle.

\section{Salle 205}

\section{GOTHIQUE FRANÇAIS}

La plupart des objets exposés dans cette salle, excepté les tapisseries qui ont été données par M. Maciet, font partie du legs Peyre.

Tapisseries (don Maciet). Vénus et sa cour (milieu du $\mathrm{xv}^{\mathrm{r}}$ siècle): Hercule, fond de fleurettes (commencement du $\mathrm{xv}^{\mathrm{e}}$ siècle): Bûcherons ( $\mathrm{xv}^{\mathrm{e}}$ siècle): tenture décorće de prons et de fleurettes, fond bleu (fin du $x^{\mathrm{e}}$ siecle): Jeune prince entouré de seigneurs, tapisserie tissée d'or (commencement du $\mathrm{xvI}^{\mathrm{c}}$ siècle): Buicherons ( $\mathrm{xv}^{\mathrm{e}}$ siècle. legs Peyre). 
Meubles: Deux lits du $\mathrm{xv}^{\mathrm{e}}$ siècle: coffres, stalles et crédences; tours à mettre le Saint-Sacrement, etc.

Vilrine centrale. Sculpture : Deux figures assises, en pierre, provenant de roussures $\left(\mathrm{x}_{11}{ }^{\mathrm{e}}\right.$ siècle); Christ entre den.x anges (bois, $\mathrm{xv}^{e}$ siecle); Vierges en pierre peinte ( $\mathrm{xv}^{\text {"n }}$ siecle).

Vitrines-tables: Sculptures, ferronneries, bois, étoffes.

\section{Salle 207 \\ GOTHIQUE FRANÇAIS}

La plupart des objets exposés dans cette salle proviennent du legs Peyre.

Aux murs: Tapisserie: Cavaliers, fin du $\mathrm{xr}^{e}$ siècle (don Maciet). - Boiseries: Panneaux sculptés, accoudoirs de stalles, etc. - Carreaux de faience, Champagne, $\mathrm{xuI}^{\mathrm{e}}-\mathrm{xV} \mathrm{e}$ s. (don Maciet). - Peinture: Jugement dernier; Trinité ( $x v^{e}$ siècle); quatre panneaux figurant Sainte Barbe, Saint Michel et Sainl Georges. -- Sculpture, marbre : Crucifixion de forme cintrée; Annonciation ct Nativité. - Bois : Vierges tenant l'enfant (fragments de nativités, don Maciet); retable peint, figurant l'Arrestation du Christ, la Flagellation, la Crucifivion et la Descente aux Limbes. $\left(x i v^{e} s.\right)$

\section{Salle 206}

\section{BALCON DE LA GALERIE DE PIERRE}

A ux murs: Bas-reliefs en marbre blanc provenant du tombeau de Claude de Lorraine, duc de Guise, à Joinville ( + I550), par Dominique Florentin et Jean Picard, dit Le Roux; ils figurent les exploits du duc (legs Peyre).

Sculptures : Vierges et Saints; Puits vénitien du commencement du $\mathrm{xv}^{\circ}$ siècle: Sphinx en marbre provenant de la chapelle du château de la Bâtie (legs Peyre). 


\section{Salle 208 \\ RENAISSANCE FRANÇAISE}

La plupart des objets exposés dans cette salle, excepté les tapisseries qui ont été données par M. Maciet, font partie du legs Peyre.

Tapisseries: Seigneur assis entre quatre femmes qui lui présentent des fleurs et des fruits; Arcatures et devises; Nativité, (legs Peyre); Lucréce et Tarquin; Docteurs dans un paysage; Loth et ses filles; Saint Jéròme; Dame Rhétorique: Enfant portant un écusson avec la devise: in aternum; Scène de Banquet; Berger paissant son troupeau (legs Peyre).

Meubles : Stalles décorées d'arabesques (école du Midi); stalles à trois places à rinceaux; coffres; panneaux de boiseries.

\section{Salle 212}

\section{RENAISSANCE FRANÇAISE}

La plupart des objets exposés dans cette salle font partie du legs Peyre.

Aux murs: Autel en marbre du château de la Bâtie, figurant Noé sortant de l'arche, Moïse passant la mer Rouge et David tuani Goliath. -- Retable en pierre peinte figurant une Sainte conversation, la Mort et les Funérailles de la Vierge. - Retable en bois figurant la Nativité, la Crucifixion et l'Assomption. - Boiseries.

Mcubles: Stalles.

Sculptures religieuses.

\section{Escalier Lefuel. - Palier du premier étage.}

Tapisserie du commencement du $\mathrm{xvI}^{\mathrm{e}}$ siècle figurant une scène de l'Histoire d'Alexandre (don Maciet). 
Salle 216

\section{RENAISSANCE FRANÇAISE}

La plupart des objets exposés dans cette salle font partie de la donation Peyre.

Aux murs: Tapisseries (don Naciet), panneau ì Heurettes, seigneur et dame, animaux dans un enclos. - Panneaux de bois, porte sculptée à décor en perspective.

Meubles : Bahuts, sièges.

Vitrine: Broderies françaises. Devants d'autels, ornements, ete.

Vitrine plate: Petits panneaux de bois.

sculpture: Vierge de Minnonciation en marbre (don Maciet). - Christ et Vierge.

Salle 218

\section{RENAISSANCE FRANÇAISE}

La plupart des objets exposés dans cette salle font partie du legs Peyre.

Passage entre les deux salles: Panneaux de bois ornés de rinceaux.

A u.v murs: Panneaux de bois ornés d'arabesques. Tapisserie: Deux femmes casquées au bord d'un puits.

Meubles: Bahuts, sièges.

Vitrine: Sculptures diverses de la Renaissance et bandes de tapisserie.

Salle 220

RENAISSANCE FRANÇAISE

La plupart des objets exposés dans cette salle font partie du legs Peyre. 
Dans le passage: Tapisserie décorée de grandes feuilles et de fleurs roses.

Aux murs : Boiseries provenant d'une maison de Rouen : petits panneaux en hauteur ornés de rinceaux et panneaux en largeur, ayant gardé une partie de leur polychromie et décorés de chimères affrontées au milieu d'arabesques. Panneaux de bois provenant du chàteau d'Assier, décorés de rinceaux du plus beau style et de la plus grande finesse.

Vitrine: Verrerie (don de Mime Patrice Salin).

\section{Salle 222}

\section{RENAISSANCE FRANÇAISE}

La plupart des objets exposés dans cette salle font partie du legs Peyre.

D.ms le passage: Panneaux de bois.

Aux murs: Cheminée de pierre avec plaque et landiers. - Panneaux et frises de bois sculptés. - Portraits de femmes. - Portrait de François Ier en pied. - Tapisserie : Vénus et l'Amour sous une treille au milieu d'arabesques.

Meubles : Bahuts et sièges.

\section{Salle 224}

\section{RENAISSANCE FRANÇAISE}

La plupart des objets exposés dans cette salle proviennent du legs Peyre.

Dans le passage, une porte sculptée et des panneaux de bois

Aux murs: Tapisserie de l'atelier de Fontainebleau, à rinceaux sur fond noir. - Panneaux de boiserie.

Meubles : Bahut, époque Henri IV (don Grandidier). Crédence à têtes en ronde bosse. - Table.

Vitrine: Verreries de Venise (don de $\mathrm{M}^{\mathrm{me}}$ Patrice Salin . 
Salle 226

RENAISSANCE FRANÇAISE

La plupart des objets exposés dans cette salle proviennent du legs Peyre.

Dans le passage: Porte sculptée et boiseries.

Aux murs: Tapis fleurdelysé à décor de personnages. France, commencement du xviI ${ }^{e}$ siècle. - Dessins de Lagneau.

Meubles : Bahuts de la fin du xvi ${ }^{e}$ siècle. - Tables et Sièges.

Vitrine: Cuirs gaufrés.

\section{Salle 230}

\section{LOUIS XIII ET LOUIS XIV}

Aux murs: Tapisseries Louis XIII : Sujets mythologiques (legs Peyre); tapisserie à fleurs. - Balcon peint en bleu et or provenant du château de Versailles (legs Peyre). - Peintures : Van Thulden, concert (don Maciet); dessins d'ornement et gravures. - Cheminée de marbre.

Meubles : Lit en étoffe verte, à applications;-Commode en marqueterie; - Stalles avec miséricordes figurant des têtes humaines.

\section{Salle 232 \\ LOUIS XIV}

Plafond allégorique (legs Peyre).

A ux murs: Tapisseries des Gobelins: pièce de la série des Château.x royau.x (Saint-Germain); deux pièces des Mois d'Audran. 
Boiseries provenant de Versailles et d'hôtels indéterminés. - Dessins d'ornement.

Meubles : Lits d'étoffe rouge et application. - Sièges et consoles.

\section{Salle 234 \\ GRANDE SALLE LOUIS XIV}

Aux murs: Tapisseries des Gobelins: pièce de la série de l'Histoire du Roi, la réception du nonce Chigi; pièce de l'Histoire des Dieux de Coypel, Neptune et Amphitrite.

- Dessus de porte peints, provenant du Louvre, Enfants tenant des emblèmes, sur fond d'or. - Glaces avec leurs trumeaux. - Boiseries provenant de Versailles et d'hòtels indéterminés; plusieurs sont de l'époque de la Régence.

Meubles : Tables, consoles, fauteuils et chaises, armoires, écrans, torchères, pendules, etc....

Vitrines : Coffrets de cuir gaufré, légués par IIme la baronne Nathaniel de Rothschild. - Fragments de boiseries.

Lutrin. - Buste d'homme (legs Peyre).

\section{Salle 228}

\section{BALCON DE PIERRE}

Groupes en stuc de Clodion, Trois femmes tenant des corbeilles de fleurs au-dessus de leurs têtes (legs Peyre).

Salle 221

ÉPOQUE DE LA RÉGENCE ET DE LOUIS XV

A ux murs: Cadre en bois doré contenant une tapisserie des Gobelins aux armes de France et de Navarre; tapisserie encadrée figurant des Colombes dans des fleurs ayant servi de dossier de canapé. - Peintures : portraits dhommes par Oudry, Hallé, Van Loo (legs Peyre); dessus de porte à sujets mythologiques par Lagrenée (legs Peyre). 
- Bustes du Maréchal de Villars (?) et d'un Evêque italien (legs Peyre). - Boiseries, trois panneaux peints par Lancret, le Turc, la Pélerine et la Femme au Parasol, provenant de l'hôtel de Boulogne, place Vendôme; dessus de porte italiens (don Bischoffsheim); balcon en bois bleu et or provenant du théâtre de Versailles (legs Peyre); panneaux de boiseries.

Meubles : consoles, commodes, sièges, lustres, bras de lumière, bronzes.

\section{Salle 219}

\section{ÉPOQUE DE LA RÉGENCE ET DE LOUIS XV}

A ux murs : Peintures : portrait d'homme, par Largillière; paysage italien; esquisse d'un Plafond de Louis de Boullongne pour un hôtel de Paris: nymphes par Trémollière (don Maciet). - Dessins d'ornement, aquarelles et gravures noires et en couleurs. - Boiseries.

Meubles: Bureau Louis XV (legs du Dr Maléçot); lustre en bronze; lit en bois doré aux armes des Rohan (don de Mme Gross) dans un encadrement d'alcôve. - Bronzes.

Vitrine: Faïences de Rouen.

Salle 217

\section{ÉPOQUE DE LA RÉGENCE}

Aux murs : Boiseries provenant de l'ancien hôtel de l'État-major de la place de Paris, place Vendôme. - Cheminée en brèche rouge, avec glace et trumeau.

\section{Salle 215 ÉPOQUE LOUIS XV}

Aux murs: Peintures: Esquisses d'un plafond rond, figurant Saint Louis montant au ciel, exécuté par Louis de 
Boullongne pour l'hôtel des Invalides; quatre panneaux accompagnent le motif central (don Féral); dessins d'ornement, aquarelles et gravures en couleurs. - Bustes de terre cuite. - Boiseries.

Vitrines A et D : Faïences de Moustier (en partie don Maciet).

Vitrine B : Faïences du Midi (en partie don I.e Secq des Tournelles).

Vitrine C : Faïences de Marseille.

Vitrines centrales $\mathbf{E}$ : Robes de dame et d'enfant.

bleu, style Bérain.

F : Pâte tendre de Saint-Cloud, décor

Vitrine G : Pâte tendre de Saint-Cloud et Mennecy blanche.

Vitrines-tables : Dentelles.

Salle 213

\section{EPOQUE LOUIS XV}

Aux murs: Peinture: Portraits d'homme et de femme, par Tournières; dessins et aquarelles; gravures en noir et en couleur, d'après Fragonard, Baudouin, etc. - Cheminée et trumeau.

Buste d'homme, en terre cuite, par Caffieri.

Vitrine: Vêtements.

\section{Salle 211 \\ EPOQUE LOUIS XV}

Aux murs ; Boiseries; Dessins et aquarelles; gravures; papiers peints.

Buste de jeune femme, en terre cuite. - Bustes d'hommes.

Meubles : sièges divers.

Vitrine A : Faïences de Nevers et Faïences étrangères. 


$$
-38-
$$

Vitrines $\mathbf{B}$ et $\mathbf{C}$. Faïences fines blanches de Paris, dites du Pont-aux-Choux, et de Lorraine, moulées sur des pièces d'argenterie.

Vitrine D : Faïences de 1'Est, Strasbourg, Niederwiller, etc.

Vitrines centrales : Argenterie, Bronzes.

Salle 209

\section{SIECLE ETRANGER}

Aux murs: Peintures: Esquisse d'un plafond et le Charlatan, par Tiepolo (don Maciet); Scène de Comédic par Troost (école hollandaise). - Gravures anglaises en couleurs.

Vitrines A : Porcelaine de Saxe ;

- B : Pâte tendre de Tournai;

- C : Porcelaine dure étrangère : Allemagne, Angleterre. Hollande (legs Gasnault, don Audéoud, etc.). 
ENTRESOL DU PREMIER ÉTAGE 



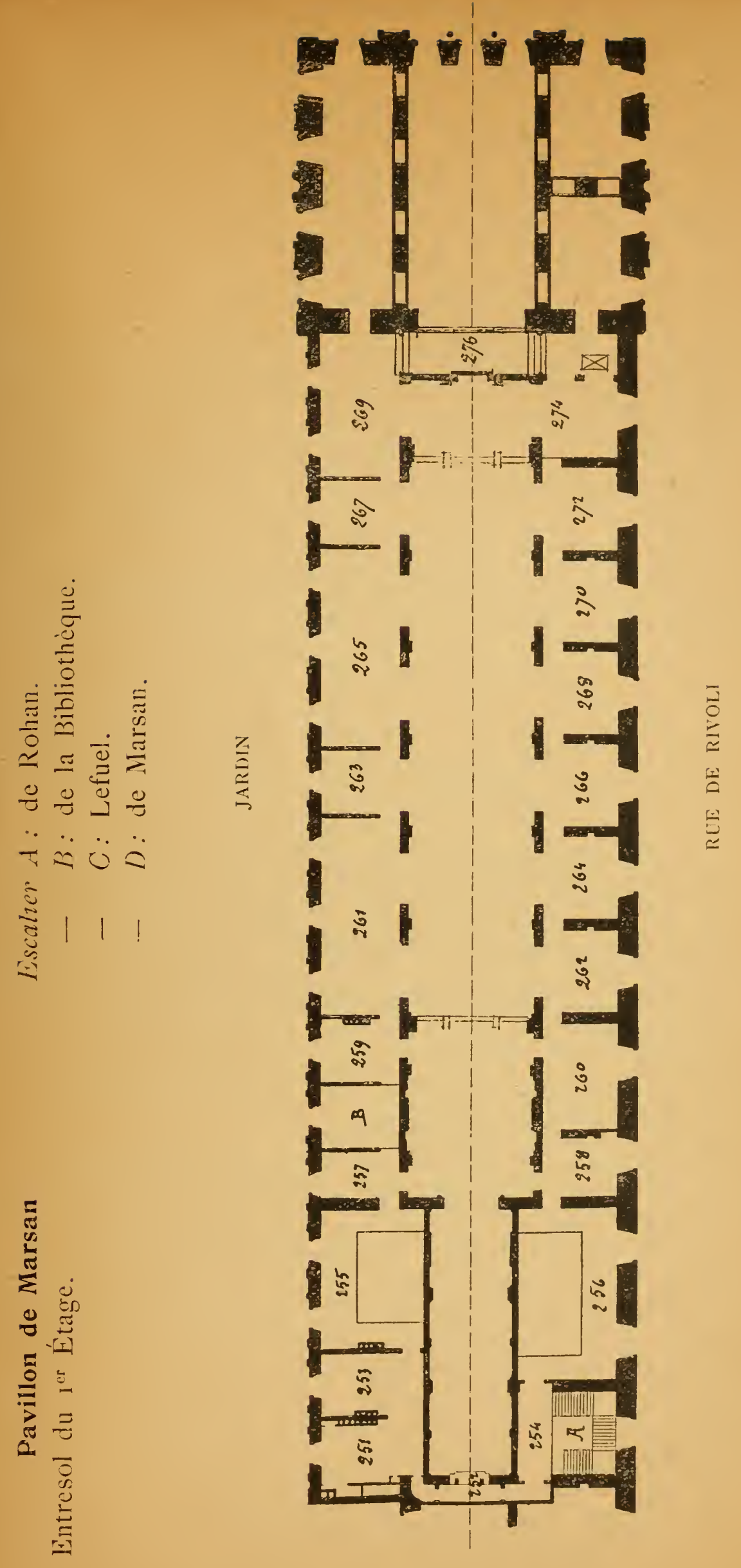




\section{Salle 259}

\section{LOUIS XVI}

Aux murs : Peintures (legs Peyre). Boilly, l'atelier de Houdon; Hubert-Robert, Banquet dans une galerie. Panneaux peints figurant les bustes des grands dramaturges au milieu de médaillons et d'arabesques - Dessins et aquarelles. - Gravures en couleurs (don Audéoud).

Meubles : Lit Directoire, en acajou. - Commode en marqueterie. - Pendules et bronzes.

Buste de l'astronome Laplace, plâtre de Houdon, le même qui est figuré dans le tableau de Boilly.

\section{Salle 261}

\section{ÉPOQUE LOUIS XVI}

A ux murs: Boiseries provenant de Versailles; entourage d'un œil-de-bœuf à décor de chiens. - Panneaux de fleurs peints par Ranson. - Gravures en couleur d'après de Bucourt, Janinet, Boilly, Lawreince; aquarelles figurant des femmes en toilette, préparation des gravures de mode de Saint-Aubin; dessins d'ornement.

Meubles : Lit de la fin du xvire siècle (don de M"le Fournier); Médaillier (don de $\mathrm{M}^{\mathrm{me}}$ la baronne de SaintPreignan); fauteuils et chaises.

Statuettes en bronze par Thomire, figurant des enfants (legs Peyre - - Pendule, consécration de la découverte des ballons (legs Peyre).

Vitrines A, B, C. Broderies, habits et robes. - D. Biscuits et terres cuites.

Vitrines-tables: Bijoux d'or (legs de $M^{\text {me }}$ la baronne Nathaniel de Rothschild). - Bronzes. 
Salle 263

\section{EPOQUE LOUIS XVI}

Petit salon orné de panneaux peints dans le style de Prieur, arabesques, fleurs et médảillons à l'antique (legs Peyre). - Cheminée en marbre blanc; console en bois doré: - pendule (legs Audéoud).

ritrine-table: Bronzes.

\section{Salle 265}

\section{ÉPOQUE LOUIS XVI}

Aux murs : Boiseries peintes en blanc provenant de Versailles, porte dorée, porte cintrée à médaillons d'amours et à guirlandes. - Panneaux peints : grisailles représentant des amours attribuées à Sauvage; fleurs de Ranson; modèles de tapisseries à fleurs pour meubles. - Modèles pour assiettes de Sèvres. - Cadre sculpté et doré, renfermant une broderie sur soie blanche figurant un aigle et léguée par M. Peyre.

Meubles : Secrétaire de Riesener, décoré de bronzes: pendule en bronze ciselé; fauteuils, chaises, etc.

Vitrines A et B : Porcelaine de Vincennes et de Sèvres: vase bleu et or, à décor de paysage et d'instruments de musique (don Gasnault); assiettes, jardinières, tête à tête, crémiers, pots à lait, etc.

Vitrine C: Porcelaine dure de Paris, Limoges, etc.

Vitrıne D : Porcelaine pâte tendre de Chantilly, Mennecy, etc.

Vitrines centrales: Biscuits, Terres cuites. 


\section{Salle 267 \\ ÉPOQUE LOUIS XVI}

Aux murs : Panneaux peints à fleurs, attribués à Leriche; panneaux de porte provenant de Versailles, à sujets turcs. - Cheminée en marbre blanc, avec trumeau en bois doré attribué à Salembier. - Chenets de bronze. - Bras de lumière à trois branches. - Gravures en couleurs, d'après de Bucourt, Huet, Baudouin (legs Audéoud).

Meubles : Fauteuils et bergères; bureau de laque de Chine; boîte à musique en bronze ciselé, ornée de cariatides.

Vitrine : Collection de moutardiers de porcelaine et de faïence du XviII ${ }^{e}$ siècle. formée par M. Ed. Hébert et donnée par M. Fitz Henry. (Sèvres, Saint-Cloud, Chantilly, Mennecy, Saxe, Rouen, etc.).

Vitrine plate: Boittes d'acier faisant partie de la collection Le Secq des Tournellès (salle 269).

\section{Salle 269}

\section{FERRONNERIE}

Collection appartenant à M. Le Secq des Tournelles.

Aux murs: Enseignes des $\mathrm{xr}^{\mathrm{e}} \mathrm{e}^{\mathrm{e}}$ et $\mathrm{xv}_{\mathrm{in}}^{\mathrm{e}}$ siècles: à SaintDenis, à l'Ermitage, à la Tourclle, à la Raquette, à la Corne d'abondance, à l'Homme armé, à la Tiare, à la Levrette; enseignes de serruriers. - Grilles: Porte de l'abbaye d'Ourscamp, près Noyon (xil ${ }^{\mathrm{e}}$ siècle). - Fenêtre de l'hôtel de Jacques Cœur à Bourges ( $\mathrm{xv}^{\mathrm{e}}$ siècle). - Grilles diverses parisiennes, surtout des $\mathrm{xvII}^{\mathrm{e}}$ et $\mathrm{xviII}^{\mathrm{e}}$ siècles.

Vitrine A : Coffrets du xrve au xvirI ${ }^{\mathrm{e}}$ siècle.

- B: Serrures

- C: Fers et porte-fers pour blanchisseuses.

- D et E: Marteaux de portes.

Vitrines de milieu: Bijouterie d'acier du xvmie siècle, boucles, peignes, châtelaines, éventails, ceintures. Outils de travail pour dames. 


$$
-4^{\overline{5}}-
$$

Vitrines plates: Clés, bonbonnières, pommes de canne, étuis et cachets, verrous, couteaux.

\section{Salle 276}

A u.x murs : Dessins de Huet pour servir de modèles à la manufacture de toiles de Jouy (don de MI. Barbet de Jouy).

\section{Salle 257}

ITALIE

Vitrine : Bronzes italiens: mortiers, coupes et figures (xv -xvie siècle). Legs Peyre.

\section{Salle 255 (balcon)}

\section{ITALIE}

La plupart des objets garnissant cette salle font partie du legs Peyre.

Fragments d'architecture en marbre. - Statuette de la Vierge tenant l'Enfant Jésus, marbre, atelier de Jean de Pise; Vierge de marbre, assise sur une chaise à dossier de mosaïque (xive siècle). - Tapisserie du xvi" siècle à fond jaune, médailles arabesques. - Peinture : Énée et Di.ton (:) (école ombrienne du xve siècle). Stucs.

\section{Salle 253}

\section{ITALIE}

La plupart des objets garnissant cette salle font partie du legs Peyre.

Faience émaillée : Vierge de Pitié entre deux saints (don de Mme Edouard André). - Peinture : Le Christ en croix entre la Vierge et Saint Jean (école de Ferrare): Sainte conversation. ( $\mathrm{rv}^{\mathrm{e}}$ siècle). - Statue funéraire (coupée) d’une femme, marbre (don Maciet); bas-reliefs en terre cuite: Deux saintes (don Maciet). - Stucs figurant La 
Vierge el l'Enfant (Florence, $x v^{e}$ siècle). - Écusson décoratif en marbre daté de 1497 . - Tapisserie : La rencontre à la porte dorée, d'après Durer, Italie, 1510. Coffres.

\section{Salle 251}

\section{ITALIE}

La plupart des objets garnissant cette salle font partie du legs Peyre.

Retable gothique : la Vierge entourée de saints, daté de i398. - Huit panneaux siennois : Histoire d'un Saint $\left(\mathrm{xv}^{\mathrm{e}}\right.$ siècle). - Coffres et devants de coffres peints, sculptés, gravés, en pâte dorée, incrustés d'ivoire ( $\mathrm{xv}^{\mathrm{e}}$ et $\mathrm{xvI}^{\mathrm{e}}$ siècle). - Berceau en bois doré (xvi ${ }^{e}$ siècle). - Statues funéraires gisantes en bois, d'homme et de femme. (Don Maciet; $\mathrm{xv}^{\mathrm{e}}$ siècle.) - Tapisseries datées de 1510 d'après Durer: Jésus parmi les Docteurs.

Dans un cadre de bois doré, tapisserie figurant César recevant la téte de Pompée.

Vitrine: Étoffes italiennes tissées.

\section{Escalier de Rohan. - $3^{\text {e }}$ pallier 254}

Aux murs: Tapisseries italiennes du $\mathrm{xvI}^{\mathrm{e}}$ siècle à fond jaune, décorées d'arabesques et de sujets mythologiques. (Legs Peyre.)

\section{Salle 256 (balcon)}

\section{ALLEMAGNE}

La plupart des objets exposés dans cette salle font partie du legs Peyre.

Sculpture: Visitation ( $\mathrm{xv}^{\mathrm{e}}$ siècle); St-Jean (Saxe ? $\mathrm{xv}^{\mathrm{e}}$ siècle); la Vierge et St-Jean (xive siècle); écusson décoratif en marbre (xve siecle). - Peinture : Adam et Eve 
( $\mathrm{xvI}^{\mathrm{e}}$ siècle); Vénus etl'A mour, atelier de Cranach ( $\mathrm{xvI}^{\mathrm{e}}$ siècle). - Meubles : Armoire alsacienne ( $\mathrm{xvl}^{\mathrm{e}}$ siècle), don Engel Dollfus; table de changeur à sujets sculptés: briques de poèle en faïence verte.

Salle 258

GOTHIQUE ESPAGNOL

La plupart des objets exposés dans cette salle font partie du legs Peyre.

Aux murs : Retable en bois peint et doré figurant des scènes de la vie de saint Jean. - Retables en bois peint des xil $^{c}$ et xim $^{e}$ siècles. - Statues. - Boiseries.

Vitrine plate : Collection de têtes de clous servant au revêtement des portes.

Salle 260

RENAISSANCE ESPAGNOLE

Aux murs: Boiseries et peintures. - Carreaux de faience. - Meubles : Bahuts ornés de plaques d'acier.

Vitrines: Étoffes et broderies espagnoles dont la plupart proviennent du legs Albert Bossy.

Salle 262

\section{ÉTOFFES GOTHIQUES (XVe \& XVI $\left.\right|^{e}$ SIÈCLE)}

Vitrine's A et B : Velours tissés d'or et brocarts italiens et flamands d'influence orientale. - Chape de velours rouge. - Broderies italiennes, allemandes et anglaises.

Salle 264

\section{ÉTOFFES DU XVIP SIÈCLE}

Vitrine A : Devants d'autel, ornements d'église, velours, soies et brocarts.

Vitrince B : Même série. 
Salle 266

\section{ÉTOFFES ITALIENNES}

Vitrine A : $\mathrm{XVI}^{\mathrm{e}}$ siècle italien, même série.

Vitrine B : $\mathrm{xvII}^{\mathrm{e}}$ siècle italien.

\section{Salle 268}

\section{ÉTOFFES FRANÇAISES}

Vitrine A : Velours et soies brochées de l'époque de Louis XIV et de la Régence.

Vitrine B : Velours et soies brochées de l'époque de Louis XV.

A ux. murs : Dessins de Huet pour servir de modèles aux toiles de Jouy, don de M. Barbet de Jouy.

Salle 270

\section{ÉTOFFES FRANÇAISES}

Vitrine A : Velours et soies de l'époque de Louis XV.

Vitrine B : Velours et soies de l'époque de Louis XVl.

Salle 272

\section{ÉTOFFES FRANÇAISES}

Vitrines A et B : Soieries et velours français de Lyon et des autres fabrications du xix ${ }^{\mathrm{e}}$ siècle.

\section{Escalier de Marsan}

Palier de l'entresol du ${ }^{\text {er }}$ étage.

Vitrine A : Étoffes françaises du $\mathrm{xix}^{\mathrm{e}}$ siècle.

Vitrine B : Étoffes russes. 
Gaylord PAMPHLET BINDER Syracuse, N. Y. Stockton, Colif. 


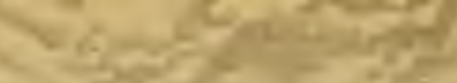

SMITHSONIAN INSTITUTION LIBRARIES

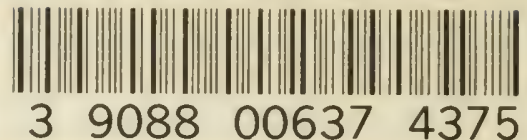

$\begin{array}{llll}3 & 9088 & 00637 & 4375\end{array}$

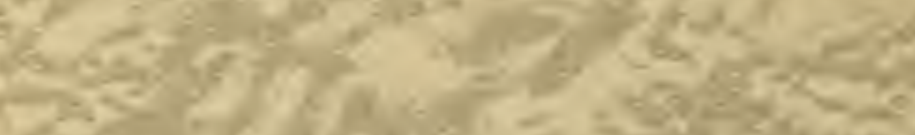

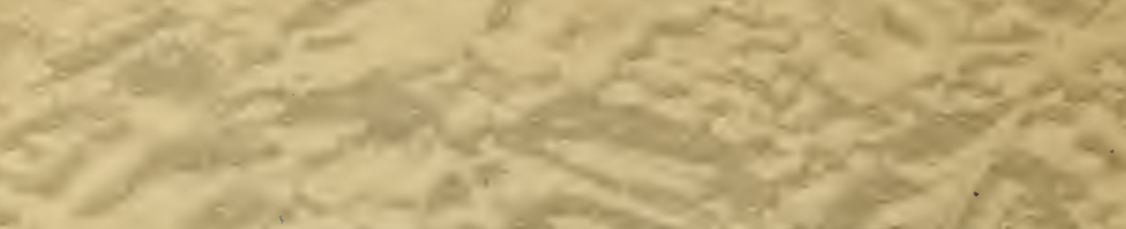
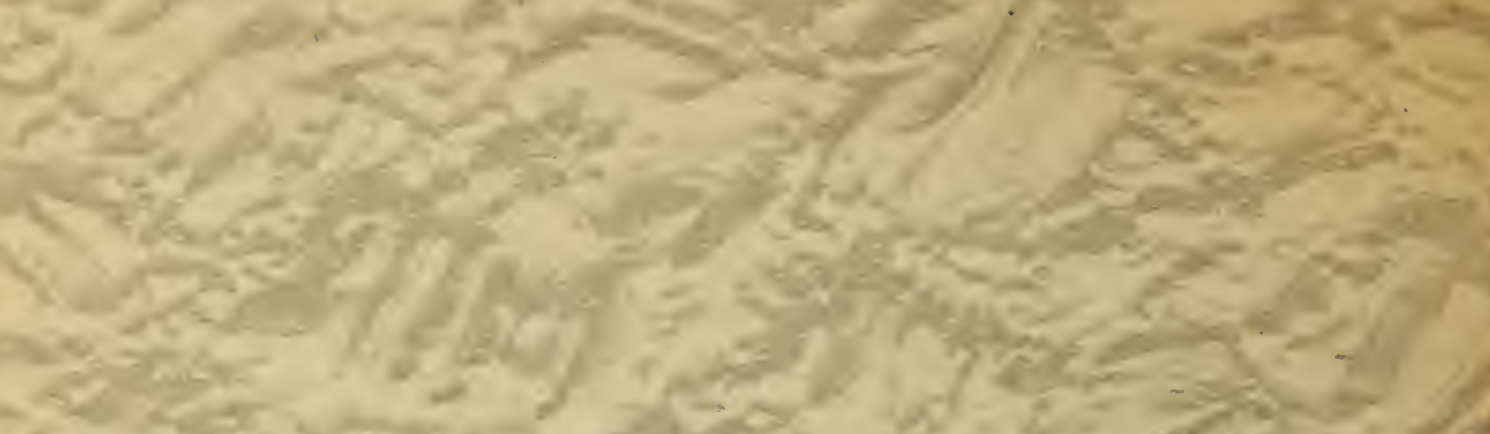

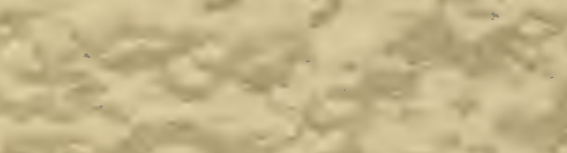

